\title{
Multidrug-Resistant Proteus mirabilis Isolated From Newly Weaned Infant Rhesus Monkeys and Ferrets
}

\author{
Wenhai Yu ${ }^{1}$; Zhanlong $\mathrm{He}^{1, *}$; Fen Huang ${ }^{2,}$ \\ ${ }^{1}$ Institute of Medical Biology, Chinese Academy of Medical Sciences, Peking Union Medical College, Kunming, Yunnan, China \\ 2 Medical Faculty, Kunming University of Science and Technology, Kunming, Yunnan, China \\ ${ }^{*}$ Corresponding author: Zhanlong He, Institute of Medical Biology, Chinese Academy of Medical Sciences, Peking Union Medical College, Kunming, Yunnan, China. Tel: +86- \\ 87168403316, E-mail: hzl@imbcams.com
}

Received: December 12, 2013; Revised: February 17, 2014; Accepted: February 28, 2014

Background: Proteus mirabilis is an important uropathogen that causes complicated Urinary Tract Infection (UTI) and induces diarrhea in infants.

Objectives: This study aimed to investigate P. mirabilis infection in newly weaned infant rhesus monkeys (Macaca mulatta) and ferrets (Mustela putorius furo) with diarrhea.

Materials and Methods: Stool samples were collected from 74 rhesus monkeys and 12 ferrets with diarrhea. Proteus mirabilis was isolated from the samples through Polymerase Chain Reaction. The isolated P. mirabilis was subjected to antimicrobial susceptibility tests.

Results: Seven $(7 / 74,9.5 \%)$ and four (4/12,30\%) P. mirabilis strains were detected in the stool samples collected from the monkeys and ferrets, respectively. Sequence analyses showed that the isolated P. mirabilis was closely related to P. mirabilis strain HI4320, which was isolated from the urine of a patient with a long-term indwelling urinary catheter. In addition, the isolates demonstrated multidrug resistance. Conclusions: Rhesus monkeys and ferrets are susceptible to P. mirabilis, making them useful as animal models for future studies on the mechanism of P. mirabilis-induced UTI and its corresponding treatment.

Keywords: Proteus mirabilis; Rhesus Monkeys; Ferrets; Diarrhea

\section{Background}

Proteus mirabilis is a pathogen that commonly causes Urinary Tract Infection (UTI) in catheterized patients and individuals with structural abnormalities of the urinary tract $(1,2)$. Proteus mirabilis uses urease to catalyze the formation of kidney and bladder stones that encrust or obstruct indwelling urinary catheters (1). Such obstructions may lead to acute pyelonephritis, fever, and bacteremia. Aside from beta-hemolytic streptococci, Escherichia coli, and Klebsiella, P. mirabilis is also a common cause of diarrhea in infants (3-5). Proteus mirabilis can also be isolated from wound infections (6). Catheter-associated UTI is a major health concern because of its complications and recurrence. However, a suitable animal model for research on P. mirabilis-induced UTI and diarrhea is lacking. Proteus mirabilis is a Gram-negative opportunistic pathogen that causes diarrhea in humans (1), cats (7), dogs $(7,8)$, and avian $(9,10)$. However, $P$. mirabilis has been rarely reported to cause spontaneous infection in rhesus monkeys or ferrets. In the present study, P. mirabilis was isolated from rhesus monkeys and ferrets with diarrhea. The isolated P. mirabilis was subjected to antimicrobial susceptibility tests. Rhesus monkey is a useful animal model for future studies on the mechanism of P. mirabilisinduced UTI and its corresponding treatment.

\section{Objectives}

The present study aimed to investigate the $P$. mirabilis infection in newly weaned infant rhesus monkeys and ferrets with diarrhea during the year 2011- 2012.

\section{Materials and Methods}

\subsection{Stool Samples}

On April 2011, 74 newly weaned infant rhesus monkeys that were housed in a nonhuman primate center suffered from acute diarrhea 1 week after weaning. Four of these monkeys died in the next 4 days. All diarrheic infant rhesus monkeys developed fever, apastia, diarrhea, bloody stool, and bacteremia. On October 2012,12 ferrets that were housed in another animal laboratory within the same center developed similar symptoms as the rhesus monkeys. Stool specimens were collected from each animal immediately to diagnose $P$. mirabilis infection based on colony morphology and Polymerase Chain Reaction (PCR) amplification. Antimicrobial tests were also performed on the isolates in preparation for treatment.

\subsection{Culture and Isolates}

The specimens were cultured in a Gram-negative enrichment broth and then incubated at $37^{\circ} \mathrm{C}$ for 12 hours. The col-

Copyright (C) 2015, Ahvaz Jundishapur University of Medical Sciences. This is an open-access article distributed under the terms of the Creative Commons Attribution-NonCommercial 4.0 International License (http://creativecommons.org/licenses/by-nc/4.0/) which permits copy and redistribute the material just in noncommercial usages, provided the original work is properly cited. 
YuW et al.

ony from the culture was inoculated on Salmonella-Shigella agar (Qingdao Hope Bio-Technilogy co., Ltd, China) and then incubated at $37^{\circ} \mathrm{C}$ overnight. On the next day, a single colony with classic colony morphology (smooth with black center) was collected from the culture plate, suspended in a nutrient broth (Qingdao Hope Bio-Technilogy co., Ltd, China), and then incubated overnight at $37^{\circ} \mathrm{C}$. DNA of the isolates was extracted using a DNA extraction kit (Axygen, China) according to the manufacturer's instructions.

\subsection{Polymerase Chain Reaction Detection}

Proteus mirabilis was detected by PCR targeting the 23s rRNA. Primers were designed according to conservative regions: P1, 5' -ACTGCGAATACCGAAGAATG-3' (433-453 nt) and P2, 5' -GAACTTACCCGACAAGGAAT-3' (1458-1478 nt, Sangon Biotech, China). The expected PCR product was 1045 bp long. The PCR conditions were as follows: $94^{\circ} \mathrm{C}$ for 3 minutes; 35 cycles of $94^{\circ} \mathrm{C}$ for 30 seconds, $58^{\circ} \mathrm{C}$ for 1 minute, and $72^{\circ} \mathrm{C}$ for 1 minute; and incubation at $72^{\circ} \mathrm{C}$ for 7 minutes. The PCR products were detected by electrophoresis on agarose gel containing $0.5 \mu \mathrm{g} / \mathrm{mL}$ ethidium bromide and sequenced using a DNA analyzer (Applied Biosystems 3730 DNA Analyzer; Invitrogen, USA).

\subsection{Sequence Analysis}

The nucleotide sequences of the amplified products and prototypes of P. mirabilis, Proteus vulgaris, Plesiomonas shigelloides, Escherichia coli, and Klebsiella pneumoniae were downloaded from GenBank and aligned using MEGA 4.0. Phylogenetic trees were generated using the minimum evolution and interior branch methods. Bootstrapping of 1,000 resampling data was performed to calculate branch percentages. The identity between nucleotide sequences was calculated using MegAlign (DNAstar package version 5.03; Lasergene, DNAstar Inc., Madison, WI, USA).

\subsection{Antimicrobial Susceptibility Test}

For susceptibility tests, the following antimicrobial agents were used: ampicillin, amikacin, norfloxacin, chloramphenicol, gentamicin, sulfamethoxazole/trimetoprim, sulfamethylisoxazole, ofloxacin, cefotaxime, and ceftriaxone sodium (Oxoid Ltd., England). The susceptibility tests were performed according to standard protocols.

\section{Results}

Seven $(7 / 74,9.5 \%)$ and four $(4 / 12,30 \%)$ P. mirabilis strains were detected in the stool samples collected from the monkeys and ferrets, respectively, through PCR and sequence analyses. Nucleotide sequences and phylogenetic analysis indicated that the P. mirabilis isolates were highly homologous (99.6\%) with the HI4320 strain (AM942759) isolated from a patient with a long-term indwelling urinary catheter (Figure 1). The P. mirabilis strains isolated from the rhesus monkeys were highly homologous with each other (97.5\% to $99.1 \%$ ) and shared $97.8 \%$ to $99.9 \%$ similarity with the strains isolated from the ferrets. This result indicated that the P. mirabilis from the rhesus monkeys infected the ferrets. The nucleotide sequences of the $P$. mirabilis strains isolated from the ferrets shared $99.0 \%$ to $99.8 \%$ similarity with each other. The results of in vitro susceptibility tests showed that the P. mirabilis strains were resistant to ampicillin, amikacin, norfloxacin, chloramphenicol, gentamicin, sulfamethoxazole/trimetoprim, and sulfamethylisoxazole but susceptible to ofloxacin, cefotaxime, and ceftriaxone sodium. Accordingly, all animals affected with diarrhea were cured 1 week after cefotaxime administration by intravenous injection.

Figure 1. Phylogenetic Analysis Based on the 23s rRNA Sequences of the 11 Isolates in This Study and 6 Other Reference Strains of Proteus mirabilis (HI4320 strain), Proteus vulgaris (AY116927), Plesiomonas shigelloides (X65487 and AL590842), Escherichia coli (FP236843), and Klebsiella pneumoniae (CP000647) Using the Neighbor-Joining Method

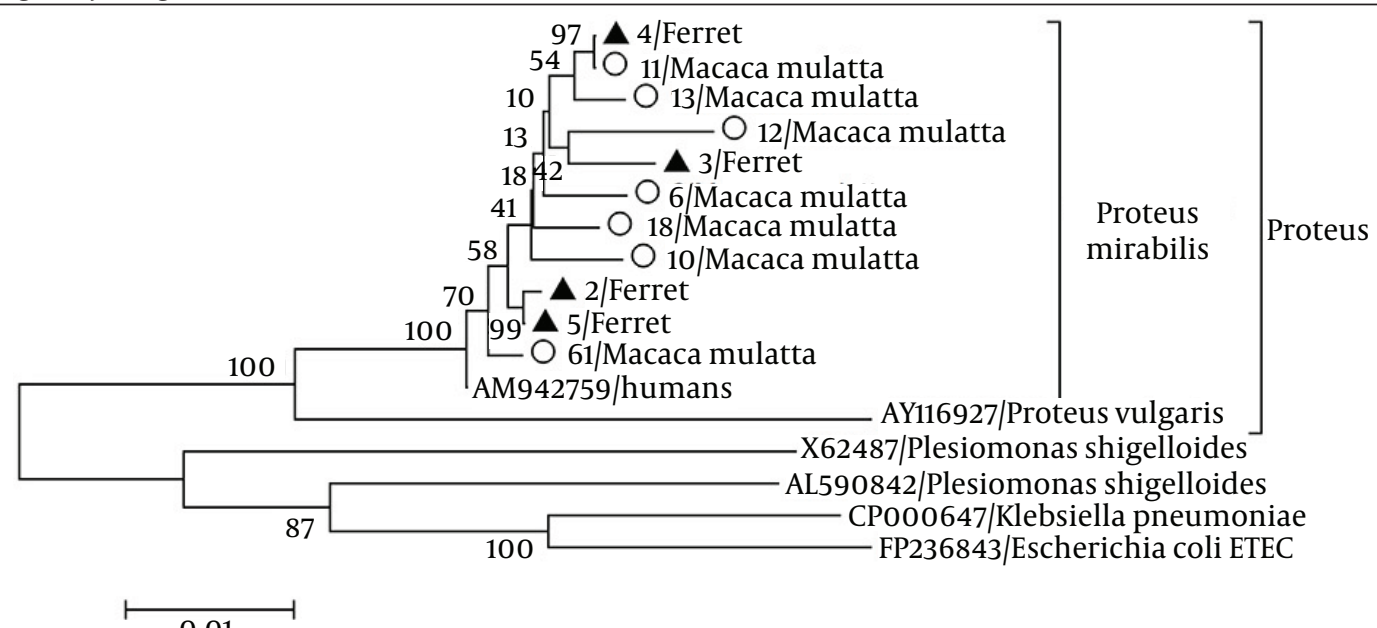

0.01

The tree was evaluated using the interior branch test method with mega 4.0. Percent bootstrap support is indicated at each node. The scale bar represents nucleotide substitutions per base. Genbank accession number and species infected with Proteus mirabilis are indicated. The isolates identified in this study are marked with triangles (Ferrets) and circles (Monkeys). 
YuW et al.

\section{Discussion}

Proteus mirabilis can be found in both healthy and diarrheic animals, as well as in food products $(7,10)$. The present study is the first to report higher infection rates of $P$. mirabilis in rhesus monkeys (9.5\%) and ferrets (30\%) than in avian (7.2\%) (9) and dogs (0.47\%) (8). The high infection rate may be caused the close contact of the same workers with all the infant rhesus monkeys. In addition, food contamination can also result in P. mirabilis infection. The transition from breast feeding to artificial feeding may have induced compromised immunity against highly pathogenic diseases in the infant monkeys and ferrets.

Urinary tract infections are among the most common bacterial infections in humans. Proteus mirabilis is an opportunistic pathogen that can cause severe UTIs, with serious kidney damages that could lead to death (11). Rhesus monkeys or ferrets are suitable animal models for future research on the mechanism of $P$. mirabilis-induced UTI and its corresponding treatment. Proteus mirabilis has emerged as a therapeutic problem because its mutations lead to resistance against antimicrobial drugs. Multidrug resistance was demonstrated by the P. mirabilis strains isolated from the rhesus monkeys and ferrets. In this study, the P. mirabilis strains were resistant to most antimicrobial drugs, except for ofloxacin, cefotaxime, and ceftriaxone sodium. The high-level of antimicrobial resistance of $P$. mirabilis isolated from food products may pose a potential threat to public health. In conclusion, the P. mirabilis strains isolated from rhesus monkeys and ferrets could facilitate future research on the mechanism of P. mirabilisinduced infection and its corresponding treatment.

\section{Acknowledgements}

We thank the Institute of Medical Biology, Chinese Academy of Medical Sciences and Peking Union Medical College for their cooperation in collecting samples and information related to this study.

\section{Authors' Contributions}

Wenhai Yu collected samples and performed detection.
Zhanlong He and Fen Huang analyzed the data and wrote the manuscript.

\section{Funding/Support}

This work was supported by the Natural Science Foundation of Yunnan Province in China (Grant No. 2011FZ068), the Fund for Talent Training for Technological Innovation of Yunnan Province in China (Grant No. 2009CI117) and Applied Basic Research Program of Yunnan Province in China (Grant No. 2013FZ142 and 2013FB032).

\section{References}

1. Pearson MM, Sebaihia M, Churcher C, Quail MA, Seshasayee AS, Luscombe NM, et al. Complete genome sequence of uropathogenic Proteus mirabilis, a master of both adherence and motility. J Bacteriol. 2008;190(11):4027-37.

2. Zunino P, Geymonat L, Allen AG, Preston A, Sosa V, Maskell DJ. New aspects of the role of MR/P fimbriae in Proteus mirabilis urinary tract infection. FEMS Immunol Med Microbiol. 2001;31(2):113-20.

3. Bi S, Yan H, Chen M, Zhang Z, Shi L, Wang H. New variant Salmonella genomic island 1-U in Proteus mirabilis clinical and food isolates from South China. J Antimicrob Chemother. 2011;66(5):1178-9.

4. Randrianirina F, Soares JL, Carod JF, Ratsima E, Thonnier V, Combe $\mathrm{P}$, et al. Antimicrobial resistance among uropathogens that cause community-acquired urinary tract infections in Antananarivo, Madagascar. JAntimicrob Chemother. 2007;59(2):309-12.

5. Spahiu L, Hasbahta V. Most frequent causes of urinary tract infections in children. Med Arh. 2010;64(2):88-90.

6. Wasfi R, Abd El-Rahman OA, Mansour LE, Hanora AS, Hashem AM Ashour MS. Antimicrobial activities against biofilm formed by Proteus mirabilis isolates from wound and urinary tract infections. Indian J Med Microbiol. 2012;30(1):76-80.

7. Hordijk J, Schoormans A, Kwakernaak M, Duim B, Broens E, Dierikx C, et al. High prevalence of fecal carriage of extended spectrum beta-lactamase/AmpC-producing Enterobacteriaceae in cats and dogs. Front Microbiol. 2013;4:242.

8. Gatoria IS, Saini NS, Rai TS, Dwivedi PN. Comparison of three techniques for the diagnosis of urinary tract infections in dogs with urolithiasis. J Small Anim Pract. 2006;47(12):727-32.

9. Awad-Alla ME, Abdien HM, Dessouki AA. Prevalence of bacteria and parasites in White Ibis in Egypt. Vet Ital. 2010;46(3):277-86.

10. Wong MH, Wan HY, Chen S. Characterization of multidrug-resistant Proteus mirabilis isolated from chicken carcasses. Foodborne Pathog Dis. 2013;10(2):177-81.

11. Pellegrino R, Scavone P, Umpierrez A, Maskell DJ, Zunino P. Proteus mirabilis uroepithelial cell adhesin (UCA) fimbria plays a role in the colonization of the urinary tract. Pathog Dis. 2013;67(2):104-7. 